INPLASY

PROTOCOL

To cite: Shu et al. Surgery plus chemotherapy versus chemotherapy alone in primary intestinal lymphoma: A Metaanalysis. Inplasy protocol 202180102. doi:

10.37766/inplasy2021.8.0102

Received: 25 August 2021

Published: 25 August 2021

Corresponding author:

Yefei Shu

shuyefei360423@163.com

Author Affiliation:

Affiliated Hangzhou Cancer Hospital, Zhejiang University School of Medicine.

Support: None.

Review Stage at time of this submission: Completed but not published.

Conflicts of interest: None declared.

\section{Surgery plus chemotherapy versus chemotherapy alone in primary intestinal lymphoma: A Meta-analysis}

\author{
Shu, Y1; Xu, X2; Yang, W3; Xu, L4.
}

Review question / Objective: Primary intestinal Lymphomas (PILs) are uncommon tumors, although time-trend analyses have demonstrated an increase. Nowadays their management is centered on systemic treatments as chemo-/radio- therapy. However, surgery is restricted to very selected indications, always discussed in a multidisciplinary setting. The aim of this meta-analysis is to evaluate the actual role of surgery in the treatment of PIL. A meta-analysis of literatures was conducted according to the recommendations of The Cochrane Collaboration.

Condition being studied: The management of primary intestinal lymphoma is controversial. Various treatment approaches have been tried, including systemic chemotherapy, primary surgical resection of intestinal lesions and postoperative chemotherapy. However, optimal treatment practices remain undefined. Among current studies, the role of surgery in intestinal lymphoma remains equivocal.

INPLASY registration number: This protocol was registered with the International Platform of Registered Systematic Review and Meta-Analysis Protocols (INPLASY) on 25 August 2021 and was last updated on 25 August 2021 (registration number INPLASY202180102).

\section{INTRODUCTION}

Review question / Objective: Primary intestinal Lymphomas (PILs) are uncommon tumors, although time-trend analyses have demonstrated an increase. Nowadays their management is centered on systemic treatments as chemo-/radiotherapy. However, surgery is restricted to very selected indications, always discussed in a multidisciplinary setting. The aim of this meta-analysis is to evaluate the actual role of surgery in the treatment of PIL. A meta-analysis of literatures was conducted 
according to the recommendations of The Cochrane Collaboration.

Rationale: We collected publications on comparison of surgery plus chemotherapy versus chemotherapy alone in PIL patients from 2000 to 2021. All trials analyzed the summary Odds Ratios (ORs) of the endpoints of interest, including 5-year overall survival rate, 3-year overall survival rate, 3-year progression-free survival rate. Revman $\mathbf{5 . 3}$ software was used to analyze the combined pooled ORs using fixed- or random-effects models according to heterogeneity.

Condition being studied: The management of primary intestinal lymphoma is controversial. Various treatment approaches have been tried, including systemic chemotherapy, primary surgical resection of intestinal lesions and postoperative chemotherapy. However, optimal treatment practices remain undefined. Among current studies, the role of surgery in intestinal lymphoma remains equivocal.

\section{METHODS}

Search strategy: We conducted a comprehensive literature search of PubMed, EMBASE, Google scholar, Cochrane Library and Clinical trial databases from January 2000 to January 2021. PIL is a relatively rare disease and therefore a 21-year timescale of [2000current] was chosen to include significant contemporary studies. If this timescale produced insufficient papers, then the period was extended to include historical papers. The search was performed by using the terms "primary intestinal lymphoma" OR "primary small intestinal lymphoma" OR "primary small bowel lymphoma" OR "primary colonic lymphoma" OR "primary large intestine lymphoma", "surgery" OR "operation", and "chemotherapy" in English-language publications (including abstracts). In addition, we hand-searched bibliographies of reviews, original studies, and relevant conference articles and contacted some investigators directly.
Participant or population: Data extraction was conducted independently by Yefei Shu and Wei Yang, and discrepancies were resolved by Xiaofeng $\mathrm{Xu}$ and Ling $\mathrm{Xu}$ before the final analysis.

Intervention: Surgery plus chemotherapy versus chemotherapy alone in primary intestinal lymphoma.

Comparator: Surgery plus chemotherapy versus chemotherapy alone.

Study designs to be included: Inclusion criteria included the following: (1) study design: the studies had to compare surgery with chemotherapy (surgical group) versus chemotherapy alone(medical group) in the treatment of intestinal lymphoma tumors; retrospective studies reporting full results of the treatment of PIL ; (2) study population: PIL patients, and the number of candidates was over 20; (3) therapy: Surgery plus chemotherapy versus chemotherapy; (4) treatment outcome reported: The main outcome outcomes (3year overall survival rate, 5-year survival rate, 3-year progression-free survival rate ) were included.

Eligibility criteria: 421 publications were identified. 324 studies were excluded following title and abstract review. The remaining 6 studies were investigated in detail and 18 studies were excluded as they did not meet the inclusion criteria for this review. All the included studies were considered to be of moderate quality at least.

Information sources: We conducted a comprehensive literature search of PubMed, EMBASE, Google scholar, Cochrane Library and Clinical trial databases from January 2000 to January 2021. PIL is a relatively rare disease and therefore a 21-year timescale of [2000current] was chosen to include significant contemporary studies. If this timescale produced insufficient papers, then the period was extended to include historical papers. 
Main outcome(s): 3-year overall survival rate, 5-year overall survival rate, 3-year progression-free survival rate.

\section{Additional outcome(s): None.}

Data management: We conducted the meta-analysis according to the recommendations of The Cochrane Collaboration and performed the statistical analysis using Review Manager 5.3 (RevMan) software.

Quality assessment / Risk of bias analysis: All included studies were graded by using the Newcastle-Ottawa Scale. The scale consists of 3 items in reporting of participants' selection, comparability of surgical group and medical group, and outcome assessment. The total quality scale was 9 points. Articles with $\geq 6$ points were considered to be of high quality.

Strategy of data synthesis: The association between surgery plus chemotherapy versus chemotherapy alone was based on data from retrospective trials. The endpoints of interest in the pooled analysis were 5-year overall survival rate, 3-year overall survival rate and 3-year progression-free survival rate, and the endpoint was considered as a weighted average of individual estimate of the OR in each included study using the inverse variance method.

Subgroup analysis: In this meta analysis, for the limited number of patients, we didn't carry out subgroup analysis based on pathology style and stage. Diffuse large B-cell lymphomas (DLBCL) account for most primary intestinal lymphomas. The 3year OS was higher in patients treated with R-CHOP (59\%) compared with CHOP $(29 \%)$. In patients with localized disease (Lugano stage I/II), surgery plus chemotherapy yielded a lower relapse rate $(15.3 \%)$ than did chemotherapy. Comparison of OS and PFS according to the treatment strategy showed no significant differences between the 2 groups in one article. For the patients with Lugano stage IV, the response and relapse rates did not differ between the 2 groups.
Sensitivity analysis: A sensitivity analysis was also performed to examine the impact on the overall results, depending on heterogeneity across the included studies. Heterogeneity was first tested using Chisquared test. A Chi-squared test with a $\mathrm{P}$ value $<0.100$ representing statistical significance. However, since tests of heterogeneity had a relative low power when there were few study we further explored heterogeneity derived from another statistical method named "inconsistency" or 12 metric, which is independent of the number of combined studies. If 12 is equal $0 \%$, there is no heterogeneity. If $12>50 \%$ heterogeneity is indicated.

Language: English-language publications (including abstracts).

Country(ies) involved: Korean, India, China.

Keywords: Surgery, chemotherapy, primary intestinal lymphoma.

Dissemination plans: None.

Contributions of each author:

Author 1 - Yefei Shu.

Author 2 - Xiaofeng Xu.

Author 3 - Wei Yang.

Author 4 - Ling Xu.

Data extraction was conducted independently by Yefei Shu and Wei Yang, and discrepancies were resolved by Xiaofeng $\mathrm{Xu}$ and Ling $\mathrm{Xu}$ before the final analysis. 